\title{
General Methods in Preparation of Skin Biopsies for Haematoxylin \& Eosin Stain and Immunohistochemistry
}

\author{
Khitam Al-Refu \\ Mutah University, \\ Jordan
}

\section{Introduction}

Cutaneous Pathology (dermatopathology) is the microscopic examination of skin biopsies in order to determine a specific diagnosis or a list of differential diagnoses. Usually the diagnosis can be determined by the routine stain for tissue sections (Hematoxylin and Eosin).

Hematoxylin is a basic stain that stains basophilic structures such as chromatin and ribosome's a deep purple or blue. Eosin is an acidic stain that stains acidophilic structures red. The advantage of this stain is that it provides a clear stain of the cell nuclei; it provides a good stain for the cell membrane. These stains are not as specific for the tissue it stains. Nonetheless, this stain aided pathologists in diagnoses.

In addition to hematoxylin and eosin, the pathologist may use additional special stains to narrow a differential diagnosis or confirm an initial impression and one of these is immunohistochemistry. Immunohistochemistry has become an important technique and widely used in many medical research laboratories and is dependent on the localization of antigens in tissue sections by the use of labeled antibody as specific reagents through antigen-antibody interactions that are visualized by a marker such as peroxidase.

\section{Skin lesion selection}

For assessment of the skin biopsies by immunohistochemistry and usual haematoxylin satin, the skin lesions which developed recently according to the patient history should be selected for that purpose. Detailed clinical information (age, sex and site of skin biopsy) of the investigated patients should be provided. Biopsies from healthy donors may be analyzed for control purposes in some cases.

\section{Types of skin biopsy}

There are four main types of skin biopsies: shave biopsy, punch biopsy, excisional biopsy, and incisional biopsy. The choice of the different skin biopsies is dependent on the suspected diagnosis of the skin lesion. Shave biopsy is done for shaving a thin layer from the top of a lesion. The punch biopsy includes the full thickness skin and subcutaneous fat and is done by using round shaped knife ranging in size from $1 \mathrm{~mm}$ to $8 \mathrm{~mm}$. In Excisional 
biopsy procedure, the doctor uses a scalpel to take off the entire lesion. Incisional biopsy is essentially the same as excisional one, except that part of lesion or tumor is included.

The skin is swabbed with an antiseptic and then injected with a local anaesthetic. In some patients, the biopsies are taken from the affected site in such a way as to include the three zones of the activity of the disease (scarred, active and apparently normal skin). Shave biopsies do not usually need stitches, while punch, excisional, and incisional biopsies will sometimes be closed with sutures.

A request form should accompany specimens that includes patient details including age, sex, site of the biopsy, brief clinical history with suspected and differential diagnosis and if there if any treatment applied prior to the biopsy.

The skin biopsies are either frozen samples or paraffin embedded. Some of the antibodies (in case of immunohistochemistry) work on frozen sections as is the case for the majority of the antibodies against the basement membrane antigens, while other antibodies such as granzyme and cutaneous lymphocytic antigen work better on paraffin sections.

\section{Preparation of paraffin embedded biopsies}

The purpose of fixation is to preserve the skin specimen indefinitely in a life-like state. For normal histological sections, the sample should be transported in fixative (usually $10 \%$ neutral buffered formalin); the volume should be at least ten times that of the tissue and should be labeled with the patient's full name, date of birth, the site of the biopsy and the date of taking the biopsy. It remains in this preservative for a minimum of 24 hours prior to processing. Formalin is a good fixative media by forming cross-links between lysine residues in the proteins of the tissues without alterations to their structure.

The biopsies are processed in the tissue processing machine for 24 hours; this machine automatically processed the tissues in graded alcohols and xylene. This involved first replacing the $70 \%(\mathrm{v} / \mathrm{v})$ ethanol with $90 \%(\mathrm{v} / \mathrm{v})$ ethanol, before placing in absolute ethanol. This is then repeated with xylene and liquid paraffin. The biopsies should not be kept in the machine for more than one week as this may affect the process of the antigen retrieval.

Skin biopsies are embedded in metal moulds filled with paraffin wax with particular attention to the position of the biopsies to ensure orientation of the tissues from the epidermis down to the subcutaneous tissues, using ice to hold the tissue in place. The cassette is positioned and the blocks are left in the ice to set. The mould is removed whilst cold and the excess wax is trimmed manually using a dissecting blade.

The wax blocks are cooled in ice and sectioned at a thickness of $4 \mu \mathrm{m}$ using a microtome. The sections are floated on a $40{ }^{\circ} \mathrm{C}$ water bath and collected on adhesive slides to minimize section loss during heat-mediated retrieval. They are then incubated at $37^{\circ} \mathrm{C}$ overnight for 24 hours on slide racks, and kept in special slides container at room temperature, to be used later on for immunohistochemistry and H\&E staining.

If the paraffin sections are not processed carefully, various artifacts may be present on the slide. Fine black spots may be seen if the formalin used is too acidic (unbuffered). In addition, holes within the sections due to tearing by microtome because of insufficient tissue dehydration or the presence of hard material such as calcium or sutures.

\section{Frozen samples preparation}

Orientation of the tissue to the knife is is an extremely important aspect of the frozen section preparation so that the knife meets the tissue in a specific orientation. Fat should be the last 
thing to hit the blade or should hit the blade by itself whenever possible. When fat hits the blade before the more manageable tissues it may smear the rest of the section.

The biopsies for frozen sections are snap frozen in liquid nitrogen, and are used directly for mounting and cutting or stored for later processing in liquid nitrogen at $-196^{\circ} \mathrm{C}$. Each biopsy is placed on a sample block within a cryostat cabinet set at a $-25^{\circ} \mathrm{C}$ and embedded in especial mounting medium, one drop is placed on the mounting plate and the biopsy is placed centrally on top. The embedding medium used for frozen tissue to ensure optimal cutting temperature; it is used to embed tissue before sectioning on a cryostat. Whilst freezing, additional drops of mounting medium fluid are added in order to completely embed the biopsy.

The frozen block (containing the biopsy) is sectioned at a thickness of $6 \mu \mathrm{m}$ and sections mounted on superfrost slides. The slides are soaked in 100\% acetone for five minutes, drained and left to air dry for 12-24 hours at room temperature. The slides are wrapped in aluminium foil, labelled and stored at $-70^{\circ} \mathrm{C}$ in liquid nitrogen. When required, the slides are removed from the freezer and allowed to stand for thirty minutes for any condensation to evaporate.

\section{Haematoxylin \& Eosin staining (H \&E)}

\subsection{Principles of H \& E staining}

H\&E stain, or hematoxylin and eosin stain, is a popular staining method in histology. It is the most widely used stain in medical diagnosis. The stain works well with a variety of fixatives and displays a broad range of cytoplasmic, nuclear, and extracellular matrix features (figure 1).

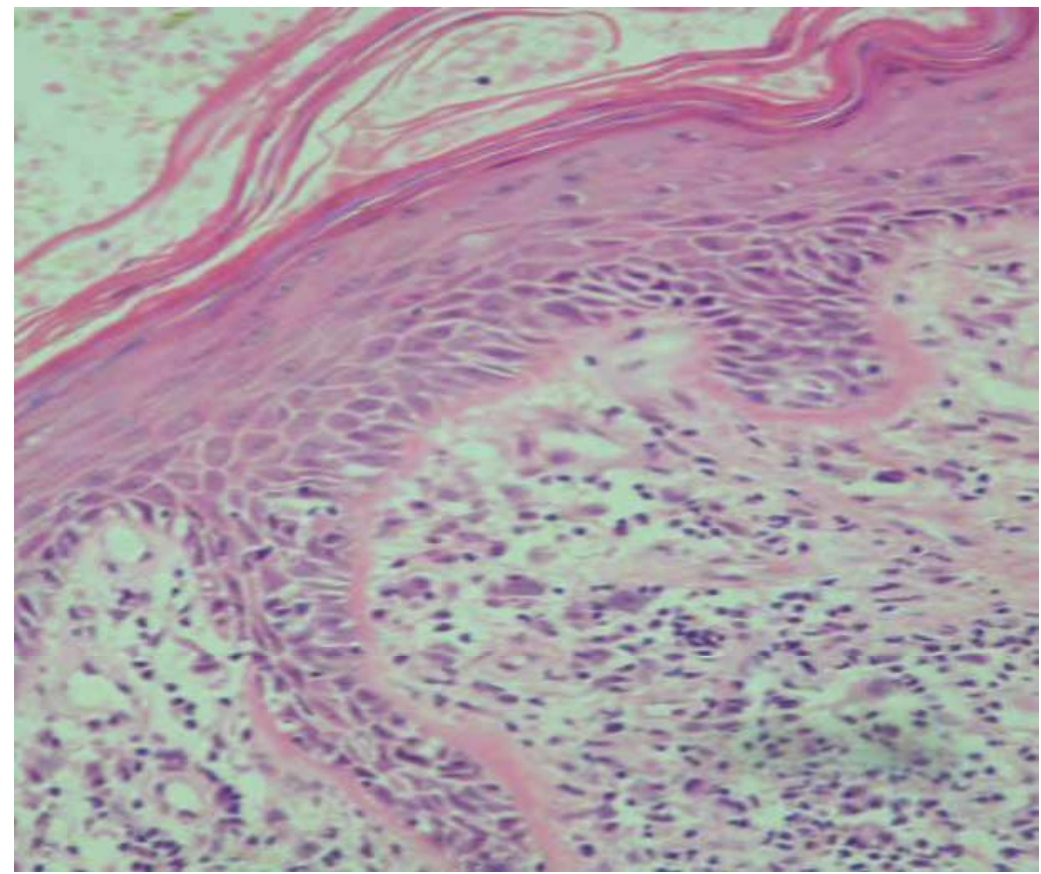

Fig. 1. Hematoxylin and eosin stain for a skin biopsy from a discoid lupus erythematosus skin lesion. 
The staining method involves application of hematoxylin, which is a complex formed from aluminium ions and oxidized hematoxylin. This colors nucleus of cells (and a few other objects, such as keratohyalin granules) blue. The nuclear staining is followed by counterstaining with eosin, which colors eosinophilic other structures in various shades of red and pink.

Optimization may be necessary to achieve staining (hematoxylin may be diluted in $\mathrm{H} 2 \mathrm{O}$ and eosin may be diluted in ethanol as needed).

\subsection{H \& E staining of paraffin and frozen sections}

Paraffin sections are prepared for H\&E staining by mounting on superfrost slides, drying on a hot plate, and then immersing into three sets of xylene for 10 minutes each followed by three sets of absolute ethanol for 10 minutes and finally rinsed with tap water. The purpose is to remove the wax and dehydrate the sections. Frozen sections are stained directly without using hot plates for drying or dehydration in sets of xylene and alcohol.

Slides (frozen and paraffin) are placed into haematoxylin for 5 minutes and rinsed thoroughly under tap water for approximately 4-5 minutes. Excess haematoxylin is removed by adding $1 \%$ acid alcohol $(1 \% \mathrm{HCl}$ in $70 \%(\mathrm{v} / \mathrm{v})$ alcohol) for 5 seconds followed by a tap water wash.

The pink haematoxylin stain is converted to blue by adding Scott's tap water, for approximately 10 seconds until the sections turned blue. The slides are rinsed in tap water before being stained in eosin $(1 \%(\mathrm{w} / \mathrm{v}))$ for 15 seconds with a subsequent wash in running tap water for 1-5 minutes. The sections are then dehydrated in two washes of absolute alcohol and in two washes of xylene for 10 minutes each before being mounted in DPX mountant and covered with glass cover slips.

\section{Immunohistochemistry by Immunoperoxidase Labelling (IHC)}

\subsection{Principles of immunohistochemistry}

Antigen-antibody interactions in tissues sections can be visualized in a number of ways. In the most common technique, an antibody is conjugated to an enzyme, such as peroxidase (immunoperoxidase staining) (figure 2), that can catalyze a color-producing reaction (see immunoperoxidase staining). Alternatively, the antibody can also be tagged to a fluorophore, such as fluorescein or rhodamine (immunofluorescence).

Immunohistochemistry or IHC refers to the process of localizing antigens in a tissue section by binding specifically with antibodies. Immunohistochemical staining is widely used in basic research to understand the distribution and localization of antigens in different parts of tissue sections.

\subsection{Retrieval of antigens from paraffin and frozen sections}

Tissues fixed in formalin (wax embedded) generally require some form of antigen unmasking for immunohistochemistry to be successful; formalin fixation and paraffin processing leads to inter and intra molecular cross linking, which mask or destroys antigenic sites in tissue specimens. Antibodies are then unable to bind them. Antigen retrieval sometimes makes the tissue act like a frozen tissue.

The most common method involves routinely heating paraffin sections in a retrieval solution, then cooling, rinsing, and proceeding with routing immunohistochemistry staining. Microwave oven, pressure cooker and steamer are the most commonly used heating methods. 
The main pitfall when performing heating methods is that extreme care must be taken not to allow the sections to dry out or overheat as this can destroy antigens and produce section artifacts. Where possible the antigen retrieval solutions were flushed from the container with cold running water, the sections could then be removed when the fluid was cool.

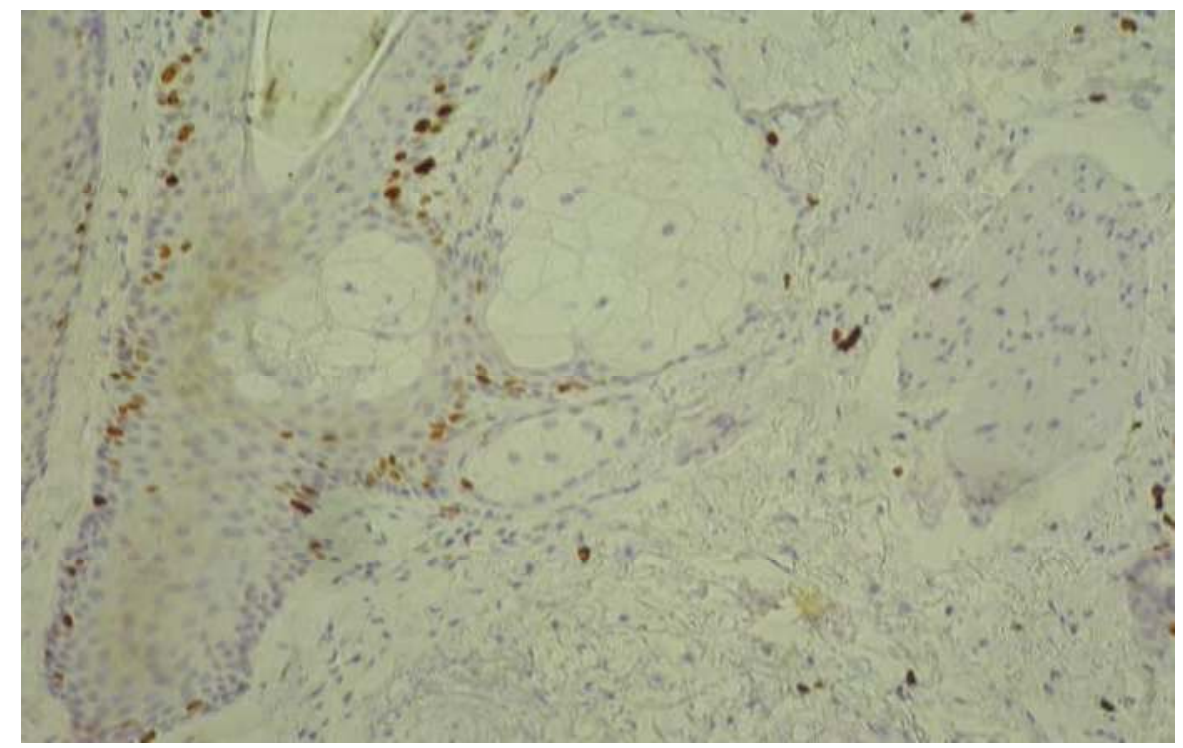

Fig. 2. Staining of skin biopsy from normal control by immunoperoxidase technique.

The type of antigen retrieval solution is different according to the primary antibody being used. The followings are commonly used Tris EDTA buffer (X10 concentrate), this solution is prepared as follows: Tris $(12.0 \mathrm{~g})$, EDTA $(1.0 \mathrm{~g}), 1 \mathrm{M} \mathrm{HCl}(10 \mathrm{ml})$ and $500 \mathrm{ml}$ of distilled water. This solution is kept refrigerated and is diluted $1 / 10$ with distilled water, and adjusted to $\mathrm{pH} 9.0$ using $\mathrm{NaOH}$ before use. Citrate buffer, this solution is prepared as follows: 2.94 g sodium citrate with $1000 \mathrm{ml}$ distilled water. The $\mathrm{pH}$ is adjusted to either 2.6 or 6.0 using $\mathrm{HCl}$. The $\mathrm{pH}$ of solutions is measured using a $\mathrm{pH}$ meter. The $\mathrm{pH}$ meter is calibrated using solutions of $\mathrm{pH} \mathrm{4,7}$ and 10 made from buffer tablets dissolved in deionised water. The $\mathrm{pH}$ of the solutions is adjusted using $1 \mathrm{M} \mathrm{HCl}$, or $1 \mathrm{M} \mathrm{NaOH}$ which is added drop-wise with stirring.

\section{Immunohistochemistry techniques}

\subsection{Sample preparation for immunohistochemistry}

A prerequisite for good immunohistochemistry is that sections (paraffin and frozen) are of the highest quality and cut from adequately fixed tissue blocks. This is critical to maintain cell morphology, tissue architecture and the antigenicity of target epitopes. Sections (frozen and paraffin-embedded) are picked up on superfrost slides to minimize section loss during heat-mediated retrieval.

De-paraffinization and rehydration of paraffin sections is required only for paraffin sections. The paraffin sections are deparaffinized in three sets of xylene for 10 minutes, each followed 
by three sets of absolute ethanol for 10 minutes and finally rinsed with tap water. The purpose is to remove the wax and rehydrate the sections. Because of the method of fixation and tissue preservation, the sample may require additional step to make the epitopes available for antibody binding, including antigen retrieval; this step often makes the difference between staining and no staining.

Additionally, depending on the tissue type and the method of antigen detection, endogenous biotin or enzymes may need to be blocked or quenched, respectively, prior to antibody staining. Endogenous peroxidase activity is found in a wide variety of mammalian tissues in red blood cells, granulocytes, monocytes, macrophages and myoglobin. The most frequently used method of blocking this endogenous enzyme activity is to use its substrate (hydrogen peroxide). Two drops of Peroxide Block (3\% Hydrogen peroxide) is used for all sections (frozen and paraffin) for 5 minutes, which are then washed twice in distilled water for 10 minutes.

Although antibodies show preferential avidity for specific epitopes, they may partially or weakly bind to sites on nonspecific proteins that are similar to the the target antigen. The nonspecific binding causes high background staining that can mask the detection of the target antigen. To reduce background staining in IHC, , the samples are incubated with a protein block that reduce the non-specific binding of primary antibodies and polymers and this done by adding two drops $(100 \mu \mathrm{l})$ of the blocking serum to the section.

The sequential steps of staining were interposed with (PBS) washing for 10 minutes.

Due to the high volume usage; PBS was made in large quantities as follows; $74 \mathrm{~g}$ sodium dihydrogen orthophosphate and $286.5 \mathrm{~g}$ disodium hydrogen orthophosphate in 25 litters of distilled water. The $\mathrm{pH}$ of this solution was 7.4.

\subsection{Antibodies types}

The antibodies (or antisera) used for specific detection can be polyclonal or monoclonal. Polyclonal antibodies are antibodies that are obtained from different B cell resources. By contrast, monoclonal antibodies are derived from a single cell line. Monoclonal antibodies show specificity for a single epitope and are therefore considered more specific to the target antigen than polyclonal antibodies. Polyclonal antibodies are a combination of immunoglobulin molecules secreted against a specific antigen, each identifying a different epitope. These antibodies are typically produced by immunization of a suitable mammal, such as a mouse, rabbit or goat and then purified from the mammal's serum. Thus, polyclonal antibodies are a heterogeneous mix of antibodies that recognize several epitopes.

\subsection{Sample labeling by immunohistochemistry}

Each section, except for the negative control, is then incubated with optimally diluted primary antibody (as recommended for each one). The optimum dilution of the primary antibody is the concentration of the primary antibody, which give the optimal specific staining with the least amount of background staining. This is determined by experiments using control sections at different concentrations and for different times. The optimal dilution is dependent upon the type and duration of fixation.

The incubation with post primary block increases penetration of the secondary reagents, the biotinylated secondary antibody was added for the slides for 30 minutes. The final incubation step involved the addition of a peroxidase conjugated avidin/biotin complex for 30 minutes. This incubation was for detection of any tissue-bound primary antibody. 
Additional of DAB working solution is required for visualization of the primary antibodies. The primary antibodies are visualized by the addition of the substrate/chromogen, 3, 3'diaminobenzidine $(\mathrm{DAB})$. This working solution isprepared by adding $50 \mu \mathrm{l}$ of $\mathrm{DAB}$ chromogen to $1 \mathrm{ml}$ of $\mathrm{DAB}$ substrate buffer and is used within 6 hours of preparation. Reaction with the peroxidase produces a brown insoluble precipitate at the antigen site. The colour development is monitored by light microscopy.

After immunohistochemical staining of the target antigen, a second stain is often applied to provide contrast that helps the primary stain stand out. Many of these stains show specificity for discrete cellular compartments or antigens, while others will stain the whole cell. A vast array of reagents to fit every experimental design, hematoxylin is commonly used. So the sections are counterstained with Haematoxylin for 5 minutes followed by rinsing in water for 5 minutes before dehydration in two sets of absolute alcohol for 10 minutes, clearing in two sets of xylene for another 10 minutes, and mounting the sections with DPX mountant and covered with glass cover slips.

\section{Development of optimal immunohistochemical methods}

There are various protocols available. Antibodies may react differently, when using antigen retrieval solutions with a different $\mathrm{pH}$. Unfortunately, there is not one method which can be applied to all antigens; some methods work with some and not others (Figure 3).

The specific staining may be too light and this may due to using too low concentration of the primary antibody (Figure 4). Or it may be too dark and this is may be due to using the primary antibody at high concentration or the incubation times may be too long (Figure 5).

\section{The controls in immunohistochemistry}

Controls validate immunohistochemistry results. Inclusion of a known positive control demonstrates the correct performance of reagents. Omission of primary antibody on one test preparation is essential. This provides information on any non-specific reactions intrinsic to the labelling system. A negative control is used on every test in which the primary antibody was omitted to test for non-specific reactions of the secondary reagents to the tissue.

\section{Double labelling protocol in immunohistochemistry}

\subsection{Principle of double labelling technique in immunohistochemistry}

In this technique, two primaries, raised in different species, are applied to the section. Two primaries visualized by different satins. Usually brown satin (DAB) for one and another satin which may be red, blue or others for the second primary. This double immune staining should not be carried out for co-stain of antigens located on the same structure (nucleus, membrane cytoplasm) as the brown stain of the DAB (the stain of the first primary antibody) combining with the blue stain (the stain of the second antibody) would give a third colour which would be difficult to differentiate from the other two colours.

Pre-request for this double immunostaing is that the secondary antibodies for both primary antibodies should be different and the antigen retrieval method in case of paraffin sections should be similar.

In peroxidase staining, the same procedure described is employed for the first antibody but without haematoxylin counterstaining. 

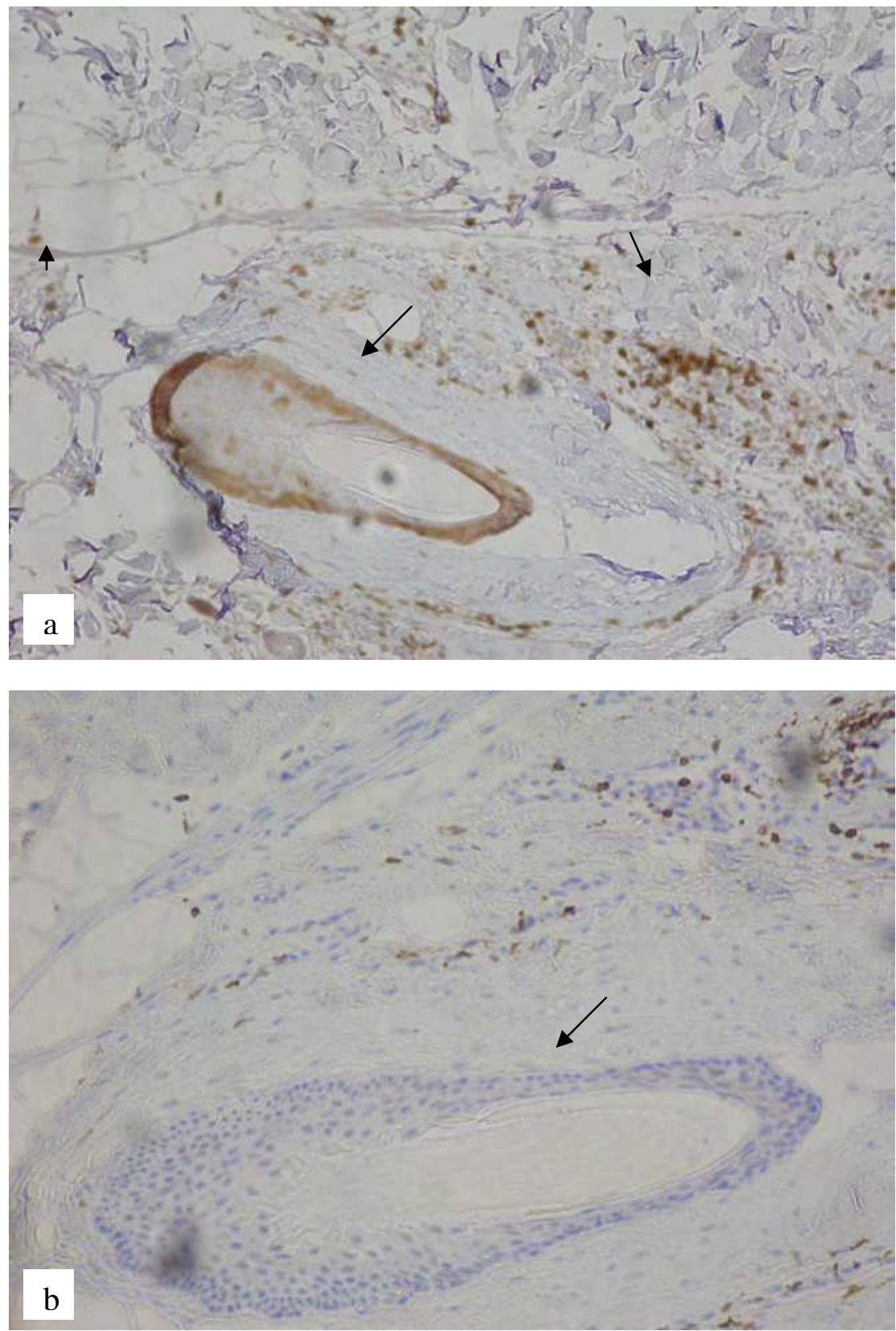

Fig. 3. This is an immunohistochemistry stain using C8/144B as a primary antibody, this can satin part of the external sheath of hair follicle and cytotoxic cells (CD8+) as demonstrated in (a) by arrows, but changing the method of antigen retrieving; the satin was very weak for the CD8 cells and without staining of the hair follicle. 

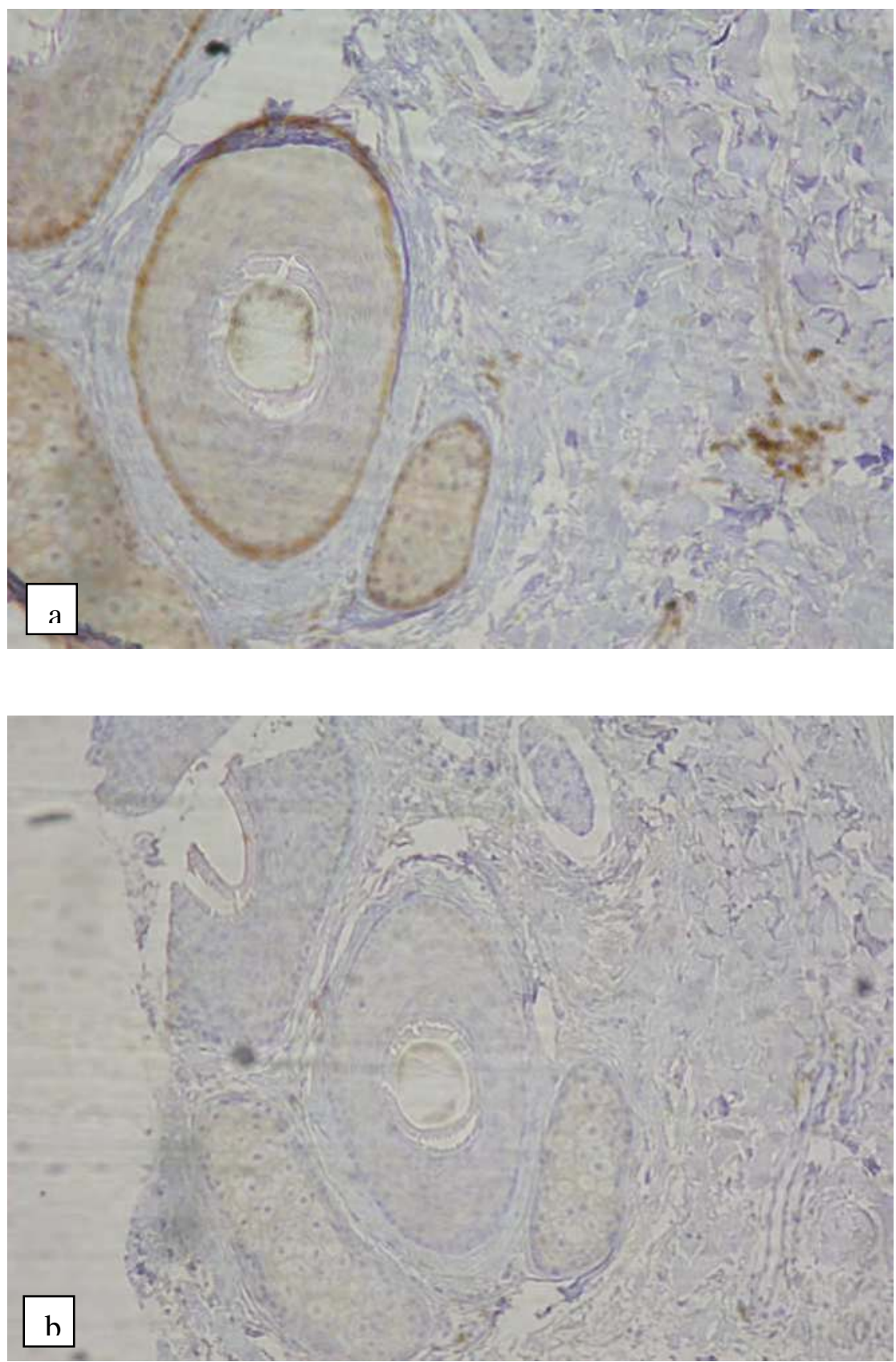

Fig. 4. This demonstrates the optimal dilution of CK-15 in (a) compared to very low concentration of this antibody in (b). 

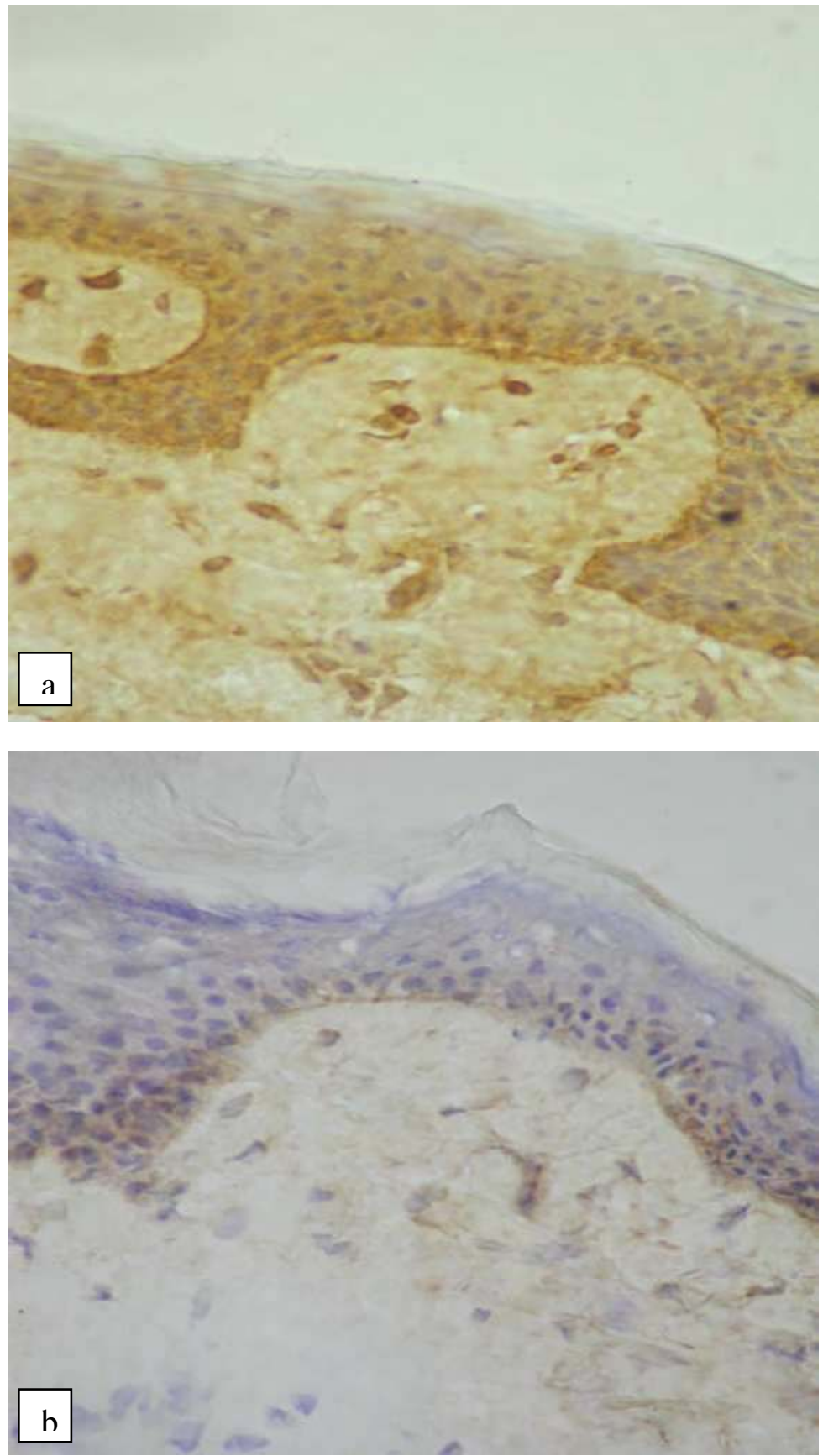

Fig. 5. This is staining with plectin as a primary antibody. (a) Expression of the plectin was normal but there was more back ground staining compared with (b). 


\subsection{Double labelling technique in immunohistochemestry}

The paraffin-embedded samples are sectioned and mounted on super adhesive slides and then de-paraffinized and rehydrated in two sets of xylene and two sets of absolute alcohol for 10 minutes. The antigen retrieval process is performed as required for both of the tested antigens. At this stage, the antigen retrieval method is similar for both of the antigens to be visualized. The endogenous peroxidase is neutralized by using peroxidase block for 5 minutes. The sections are then incubated with protein block as required for the first primary antibody followed with incubation with the optimally diluted primary antibody. This is followed by addition of the post primary block and secondary antibody as required for the tested first primary antibody. The visualization of the first antibody is carried out by DAB working solution. At this stage, there is no counterstaining with haematoxylin. The slides are then washed with BPS as usual.

Staining with the second primary antibody as described below.

\subsection{Primary staining with the second antibody}

The slides are incubated with the protein block as required for the second primary. The second antibody is added to the slides after dilution with BPS. This is followed by addition of the secondary antibody as required for the second primary antibody. After washing of slides with BPS as usual, the specific reagent for visualization of the second primary antibody is added to the sections. The blue colour is the best to be combined with the brown stain of the DAB stain. The sections are mounted with DPX mountant and covered with glass cover slides without haematoxylin staining. Using of haematoxylin staining may mask the required satin of the second antibody (which will be stained with blue colour).

\section{References}

[1] Kiernan JA (2008). Histological and Histochemical Methods: Theory and Practice. 4th ed. Bloxham, UK: Scion.

[2] Lillie RD, Pizzolato P, Donaldson PT (1976). Nuclear stains with soluble metachrome mordant lake dyes. The effect of chemical endgroup blocking reactions and the artificial introduction of acid groups into tissues. Histochemistry 49: 23-35.

[3] Ramos-Vara, JA (2005). "Technical Aspects of Immunohistochemistry". Vet Pathol 42 (4): 405-426. doi:10.1354/vp.42-4-405.

[4] Al-Refu K, Goodfield M (2009). Hair follicle stem cells in the pathogenesis of the scarring process in cutaneous lupus erythematosus. Autoimmun Rev. 8 (6):474-7.

[5] Al-Refu K, Edward S, Ingham E, Goodfield M. Br J Dermatol (2009). Expression of hair follicle stem cells detected by cytokeratin 15 stain: implications for pathogenesis of the scarring process in cutaneous lupus erythematosus 160 (6):1188-96.

[6] Al-Refu K, Goodfield M. Immunohistochemistry of ultrastructural changes in scarring lupus erythematosus (2011). Clin Exp Dermatol 36(1):63-8.

[7] Shi S - R, Cote RJ, Taylor CR (1999). Standardization and further development of antigen retrieval immunohistochemistry: strategies and future goals. J. Histotechnol 22: 177 $-192$.

[8] O' Leary TJ (2001). Standardization in immunohistochemistry. Appl. Immunohistochem. Mol. Morphol 9: $3-8$. 
[9] Scanziani E (1998). "Immunohistochemical staining of fixed tissues". Methods Mol Biol 104: 133-40. 


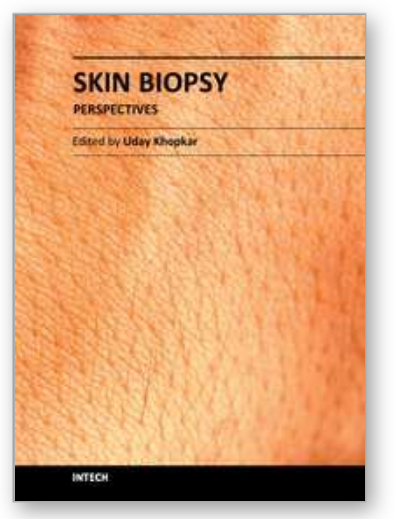

\author{
Skin Biopsy - Perspectives \\ Edited by Dr. Uday Khopkar
}

ISBN 978-953-307-290-6

Hard cover, 336 pages

Publisher InTech

Published online 02, November, 2011

Published in print edition November, 2011

Skin Biopsy - Perspectives is a comprehensive compilation of articles that relate to the technique and applications of skin biopsy in diagnosing skin diseases. While there have been numerous treatises to date on the interpretation or description of skin biopsy findings in various skin diseases, books dedicated entirely to perfecting the technique of skin biopsy have been few and far between. This book is an attempt to bridge this gap. Though the emphasis of this book is on use of this technique in skin diseases in humans, a few articles on skin biopsy in animals have been included to acquaint the reader to the interrelationship of various scientific disciplines. All aspects of the procedure of skin biopsy have been adequately dealt with so as to improve biopsy outcomes for patients, which is the ultimate goal of this work.

\title{
How to reference
}

In order to correctly reference this scholarly work, feel free to copy and paste the following:

Khitam Al-Refu (2011). General Methods in Preparation of Skin Biopsies for Haematoxylin \& Eosin Stain and Immunohistochemistry, Skin Biopsy - Perspectives, Dr. Uday Khopkar (Ed.), ISBN: 978-953-307-290-6, InTech, Available from: http://www.intechopen.com/books/skin-biopsy-perspectives/general-methods-inpreparation-of-skin-biopsies-for-haematoxylin-eosin-stain-and-immunohistochemistr

\section{INTECH}

open science | open minds

\section{InTech Europe}

University Campus STeP Ri

Slavka Krautzeka 83/A

51000 Rijeka, Croatia

Phone: +385 (51) 770447

Fax: +385 (51) 686166

www.intechopen.com

\section{InTech China}

Unit 405, Office Block, Hotel Equatorial Shanghai

No.65, Yan An Road (West), Shanghai, 200040, China 中国上海市延安西路65号上海国际贵都大饭店办公楼 405 单元

Phone: +86-21-62489820

Fax: +86-21-62489821 
(C) 2011 The Author(s). Licensee IntechOpen. This is an open access article distributed under the terms of the Creative Commons Attribution 3.0 License, which permits unrestricted use, distribution, and reproduction in any medium, provided the original work is properly cited. 